\title{
EFEITO DA REDE DE RESERVATÓRIOS SOBRE A ENERGIA HIDRÁULICA ACUMULADA EM BACIA HIDROGRÁFICA DE MESO-ESCALA NO SEMIÁRIDO BRASILEIRO
}

\author{
Antonia Tatiana Pinheiro do Nascimento, Allison Gurgel Macambira, \\ Pedro Henrique Augusto Medeiros \\ Instituto Federal de Educação, Ciência e Tecnologia do Ceará, Campus de Maracanaú \\ <tati16pinheiro@hotmail.com>, <allison.macambira@ outlook.com> \\ $<$ phamedeiros@ifce.edu.br>
}

DOI: $10.21439 /$ conexoes.v10i5.1176

\begin{abstract}
Resumo. O Nordeste do Brasil está inserido em região semiárida cujas precipitações irregulares, associadas a elevadas taxas de evaporação potencial e solos rasos, são características predominantes. Dentro deste contexto, diversas técnicas de sobrevivência surgiram, como por exemplo, a construção de reservatórios superficiais para o armazenamento de água. No presente trabalho avalia-se o efeito da rede de reservatórios sobre a energia hidráulica acumulada na bacia do açude Fogareiro $\left(7.700 \mathrm{~km}^{2}\right)$, Ceará, em diferentes cenários de armazenamento de água. Observa-se que os pequenos açudes se encontram em grande quantidade, entretanto disponibilizam conjuntamente uma menor quantidade de água quando comparada com os reservatórios estratégicos de maior porte. No entanto, esses reservatórios tendem a distribuir espacialmente a disponibilidade hídrica, o que reduz o gasto com energia elétrica para abastecimento hídrico de comunidades mais remotas. Há ainda um ganho de energia hidráulica potencial do sistema, uma vez que esses reservatórios se localizam geralmente nas cabeceiras das bacias e, portanto, em cotas mais elevadas, reduzindo-se não apenas os comprimentos das linhas de adução mas também a altura manométrica. Assim, os pequenos reservatórios promovem uma maior eficiência energética dos sistemas de distribuição de água.
\end{abstract}

Palavras-chaves: Rede de reservatórios. Disponibilidade hídrica, Eficiência energética. Semiárido.

\begin{abstract}
The Northeast of Brazil is inserted in a semiarid region where irregular precipitations, associated with high rates of potential evaporation and shallow soils, are predominant characteristics. Within this context, several survival techniques have emerged, such as the construction of surface reservoirs for the storage of water. In the present work it is evaluated the effect of the reservoir network on the hydraulic energy accumulated in the Fogareiro reservoir catchment $\left(7,700 \mathrm{~km}^{2}\right)$, Ceará, Brazil, in different water storage scenarios. It is observed that the small reservoir are present in large quantity, however they jointly offer a smaller quantity of water when compared with the larger strategic reservoirs. Nonetheless, these reservoirs tend to spatially distribute the water availability, which reduces the amount of electricity used to supply water to more remote communities. There is also a potential hydraulic power gain of the system, since these reservoirs are generally located in the headwaters of the basins and, therefore, in higher altitudes, reducing not only the lengths of the adduction lines but also the manometric height. Thus, small reservoirs promote greater energy efficiency of water distribution systems.
\end{abstract}

Keywords: Reservoir network. Water availability. Energetic efficiency, semiarid.

\section{INTRODUÇÃO}

$\mathrm{O}$ crescimento populacional associado a altas taxas de consumo de água per capita têm aumentado a demanda hídrica, causando problemas principalmente nas regiões áridas e semiáridas, onde a escassez hídrica é uma realidade (ADHAM et al. 2016). Segundo Rocha e Soares 
EFEITO DA REDE DE RESERVATÓRIOS SOBRE A ENERGIA HIDRÁULICA ACUMULADA EM BACIA HIDROGRÁFICA DE MESO-ESCALA NO SEMIÁRIDO BRASILEIRO

(2015), essas regiões abrangem 54\% da área agrícola onde vivem cerca de um bilhão de pessoas. O Nordeste do Brasil (NEB) está inserido em região semiárida cujas precipitações irregulares com média de 700 $\mathrm{mm}$ /ano, associadas a elevadas taxas de evaporação potencial de aproximadamente $2.500 \mathrm{~mm} /$ ano e solos rasos, são características predominantes (DOS SANTOS et al. 2009; DE ARAÚJO; GÜNTNER; BRONSTERT 2006). A natureza é adversa, fortemente influenciada pela seleção natural e adaptada a resistir às periódicas fases de estresse hídrico e a suprir os excessos em períodos de cheias (PAIVA, 2008). Essas particularidades têm consequência direta na sobrevivência do homem nessas regiões, pois impõem a necessidade de técnicas adequadas à convivência com a escassez hídrica (ANDRADE; PEREIRA; DANTAS, 2010).

Dentro desse contexto, diversas políticas têm sido sugeridas e aplicadas para lidar com a escassez de água e para mitigar os impactos negativos causados pelas secas para os diferentes usos (abastecimento humano, irrigação, indústria), dentre elas estão incluídas a alocação da água, reuso, aproveitamento da água da chuva (??). A construção de reservatórios tanto pelos governos estaduais e federal, quanto por moradores do NEB é a técnica mais utilizada, resultando em uma densa rede de pequenos, médios e grandes reservatórios. Estimase que na década de 90 existia algo em torno de 70.000 açudes no Nordeste do Brasil (SILANS, 2003).

Malveira, Araújo e Güntner (2012) realizaram levantamento dos reservatórios da bacia hidrográfica do Alto Jaguaribe $\left(24.200 \mathrm{~km}^{2}\right)$ e identificaram aproximadamente 4.500 reservatórios, resultando em uma área de contribuição de aproximadamente $5,5 \mathrm{~km}^{2}$ por açude.

O processo de acumulação de água nos reservatórios é totalmente dependente do regime de vazões na região, cuja variabilidade temporal é elevada. A água é armazenada nos períodos de cheia e é retirada no período de estiagem (PETER et al., 2014). Portanto, para a população local, os pequenos reservatórios são a fonte de água mais importante para lidar com a seca, promovendo, por exemplo, a diversificação da produção agrícola (LIEBE et al. 2009, FOWE et al., 2015).

No entanto, a implantação indiscriminada dos pequenos açudes pode causar impacto na disponibilidade hídrica de grandes reservatórios através do aumento das perdas por evaporação, além de comprometer a quantidade e a qualidade da água (LIMA NETO; WIEGAND; ARAÚJO, 2011; KROL et al., 2011). Segundo Callow e Smettem (2009), os pequenos açudes, de forma individual, pouco interferem nas variáveis hidrológicas. Todavia, os mesmos autores salientam que o seu efeito cumulativo pode ser considerável dentro das conectividades hidráulica e sedimentológica.

Água e energia são consideradas fatores chaves para a geração de renda, desenvolvimento social e qualidade de vida (FERREIRA et al., 2016). O aumento no consumo de energia devido ao crescimento populacional e o considerável avanço das tecnologias têm despertado a atenção do mundo para a produção de energias limpas. Isso resulta em uma maior contribuição destas fontes nos últimos anos e sinaliza o crescimento para o futuro próximo (??).

Nas redes de distribuição de água, a energia é a principal responsável pelo transporte, desde sua captação nos reservatórios, tratamento nas Estações de Tratamento de Água (ETA) até chegar ao consumidor final. Cada litro de água que se move pelo sistema representa um significativo custo de energia (ALBUQUERQUE; FIRMINO; CURI, 2004). Segundo o Ministério de Minas e Energia, os sistemas de abastecimento de água consomem aproximadamente 10.000.000 MWh/ano (BRASIL, 2011). Em porcentagem, o consumo nacional de eletricidade, destinado ao setor de tratamento e distribuição de água e esgotos é de 3\% (PROSAB, 2009).

A construção de reservatórios de pequeno porte tende a distribuir espacialmente a disponibilidade hídrica, o que reduz o consumo com eletricidade para estes setores (KROL et al., 2011). Portanto, as redes que possuem reservatórios de vários tamanhos devem apresentar maior eficiência hidrológica e energética, podendo-se inferir que, caso não houvesse pequenos e médios açudes, a água armazenada apresentaria cotas mais baixas (armazenando-se nos reservatórios estratégicos), demandando mais energia para disponibilizá-la para as comunidades a montante, localizadas em cotas mais elevadas (DE ARAÚJO; MEDEIROS, 2013). Dentro desse contexto, o presente artigo tem como objetivo avaliar o efeito da rede de reservatórios sobre a energia hidráulica acumulada na bacia do açude Fogareiro $\left(7.700 \mathrm{~km}^{2}\right)$, Ceará, em diferentes cenários de armazenamento de água, ou reservação.

\section{MATERIAIS E MÉTODOS}

\section{1 ÁREA DE ESTUDO}

O Açude Antônio Ferreira Antero (Fogareiro, Figura 1. tem capacidade de aproximadamente $119 \mathrm{hm}^{3}$, drenando uma área de $7.700 \mathrm{~km}^{2}$ na bacia hidrográfica do Banabuiú, Ceará. O reservatório está localizado no município de Quixeramobim, sertão central, sob as coordenadas em $5^{\circ} 10,0^{\prime} \mathrm{S} 39^{\circ} 29,5^{\prime} \mathrm{W}$. A precipitação média é de aproximadamente $750 \mathrm{~mm} / \mathrm{ano}$, com eva- 
EFEITO DA REDE DE RESERVATÓRIOS SOBRE A ENERGIA HIDRÁULICA ACUMULADA EM BACIA HIDROGRÁFICA DE MESO-ESCALA NO SEMIÁRIDO BRASILEIRO

poração potencial média da ordem de $2.000 \mathrm{~mm} / \mathrm{ano}$ e índice de aridez 0,35 (DE ARAÚJO; PIEDRA, 2009. FUNCEME, 2008). A classificação climática de Köppen define o clima como semiárido-'Bs' (GAISER et al. 2003).

\subsection{MODELO DIGITAL DO TERRENO}

Foram utilizados dados SRTM (Shuttle Radar Topography Mission) com resolução espacial de $90 \mathrm{~m}$ para elaboração de modelo digital do terreno, cobrindo toda a bacia hidrográfica do açude Fogareiro. A partir de processamento dos dados utilizando-se o software ArcGIS 10.2, foram obtidas a altitudes dos reservatórios superficiais presentes na bacia e com área de inundação superior a 5 ha. O mapeamento dos reservatórios foi realizado pela Fundação Cearense de Metrologia e Recursos Hídricos (FUNCEME, 2008), tendo sido utilizado neste estudo o produto final do mapeamento, disponibilizado na forma de arquivo com informações geoespacias na forma de vetores (shapefile).

\subsection{LEVANTAMENTO DE DADOS DOS RESERVA- TÓRIOS E SUA CLASSIFICAÇÃO}

Verificou-se a necessidade de estimar os volumes de cada reservatório presente na bacia hidrográfica do açude para sua classificação e cálculo da disponibilidade hídrica. A estimativa da capacidade do reservatório a partir da área inundada requer a utilização de duas variáveis, o coeficiente de abertura do reservatório $(\mathrm{K})$ e o coeficiente de forma $(\alpha)$, consideradas constantes para a referida região. Pereira (2014) estimou K equivalente a 2002 e $\alpha$ equivalente a 2,77 para a bacia do Banabuiú. O cálculo do volume $\left(\mathrm{V}, \mathrm{em} \mathrm{m}^{3}\right)$ a partir da área máxima obtida do levantamento da FUNCEME $\left(\mathrm{A}\right.$, em $\left.\mathrm{m}^{2}\right)$ dos reservatórios se deu pela equação 1

$$
V=\left(\frac{A}{\alpha K}\right)^{\frac{\alpha}{\alpha-1}}
$$

Com suas capacidades definidas, os reservatórios localizados na bacia do açude Fogareiro foram classificados de acordo com suas respectivas capacidades e, posteriormente, inseridos em uma das classes definidas: até $0,5 \mathrm{hm}^{3} ; 0,5$ a $3,5 \mathrm{hm}^{3} ; 3,5$ a $20 \mathrm{hm}^{3}$; e a última classe composta somente pelo açude Fogareiro.

A rede de reservatórios em estudo é representada através de modelo cascata, no qual se verifica a capacidade de armazenamento de cada reservatório, classificando-os e agrupando-os em classes. Esse modelo admite que os reservatórios de classes inferiores, que possuem menor capacidade de armazenamento, estão à montante dos reservatórios de classes superio- res, que possuem maior capacidade (GÜNTNER et al. 2004). Nesse modelo, o fluxo de água é transferido de cada classe para as classes subsequentes, representando as conexões entre os reservatórios quando da ocorrência de extravasamento dos reservatórios, ou vertimento

\subsection{BALANÇOS HÍDRICOS}

O cálculo do balanço hídrico foi realizado através da equação 2, o que possibilitou calcular a retirada de água possível dos açudes pertencentes à bacia do reservatório estratégico. A simulação foi realizada para um reservatório representativo de cada classe, admitindo-se que todos os outros sejam semelhantes. Os componentes do balanço hídrico foram estimados em escala mensal a fim de se obter os valores da vazão regularizada para cada classe considerada.

$$
V_{r+1}=V_{t}+P+E s c-(E V+R+V e r t),
$$

em que: $V_{t+1}$ é o volume total de água armazenado no fim do mês $\left(\mathrm{m}^{3}\right)$, $V_{t}$ é o volume total de água armazenado no início do mês $\left(\mathrm{m}^{3}\right), P$ é a precipitação mensal sobre o espelho d'água do açude $\left(\mathrm{m}^{3}\right), E s c$ é o volume estimado de água escoada na bacia hidrográfica no mês $\left(\mathrm{m}^{3}\right), E V$ é o volume mensal de água evaporado do reservatório $\left(\mathrm{m}^{3}\right), R$ é o volume mensal de água retirado para uso $\left(\mathrm{m}^{3}\right)$, também conhecido como vazão regularizada, Vert é o volume mensal de água vertido $\left(\mathrm{m}^{3}\right)$. Em geral, a infiltração e a recarga no reservatório, que também seriam passíveis de entrar no cálculo, são consideradas desprezíveis no Nordeste do Brasil, devido às condições geomorfológicas da região.

Considerou-se uma garantia de disponibilidade de água de 90\% (Q90) para cálculo da vazão regularizada, a qual foi calculada levando em conta as falhas que ocorriam no sistema. Por falhas, compreende-se que o volume de água a ser retirado em um determinado mês não foi efetivado, devido ao fato de que o volume do reservatório não seria capaz de suprir a quantidade necessária. Ou seja, uma garantia de $90 \%$ representa uma probabilidade de falha de $10 \%$ no abastecimento.

\section{RESULTADOS E DISCUSSÕES}

Verifica-se na Figura 2 que os açudes de menor porte tendem a apresentar altitudes mais elevadas, o que confirma a hipótese proposta por Güntner et al. (2004) e adotada neste estudo de que, quando se analisa o efeito cascata de um sistema, os reservatórios de menor porte estão a montante.

Uma análise por faixas de altitudes indica que, no intervalo de 235 a 335 metros, além do reservatório es- 


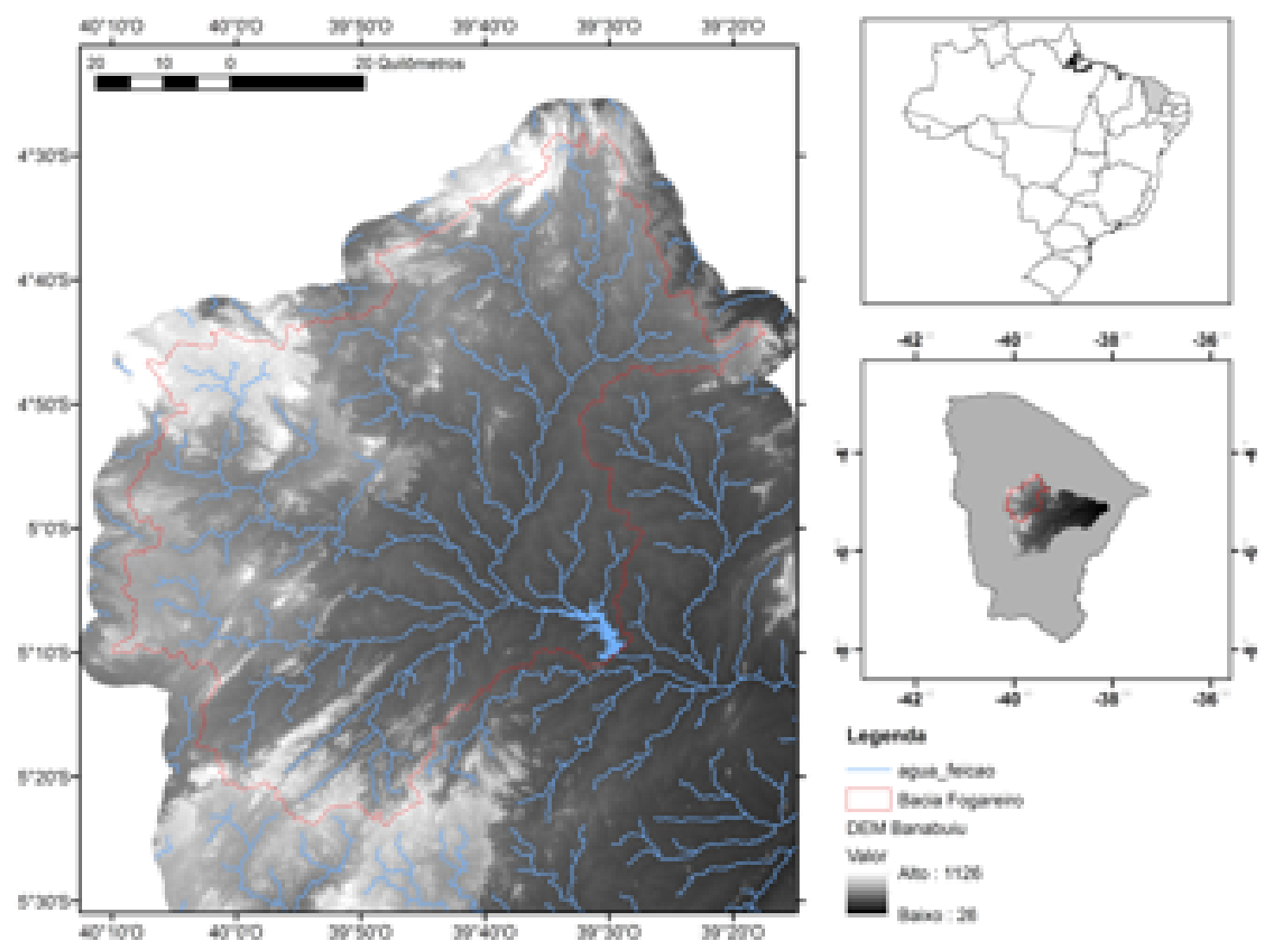

Figura 1: Mapa de localização da bacia hidrográfica do Açude Fogareiro 
tratégico existem também 127 açudes de diversos tamanhos, representando $52,7 \%$ da quantidade de reservatórios presentes na bacia para o cenário atual. O volume total de água reservada nessa faixa de altitudes equivale a $269,1 \mathrm{hm}^{3}$, enquanto que a capacidade de acumulação da bacia como um todo é igual a $311,7 \mathrm{hm}^{3}$. Ou seja, a faixa de altitudes correspondente aos 100 metros mais baixos representa $86,3 \%$ da capacidade de acumulação de água da bacia, já que o reservatório principal de maior volume está incluso nessa faixa.

Para o intervalo de cotas que variam de 435 a $535 \mathrm{~m}$, observa-se a presença de 32 reservatórios, ou 13,3\% do total. Já o volume de água armazenado na bacia, para essa faixa de altitude, cai para $9,2 \mathrm{hm}^{3}$, ou aproximadamente $3 \%$ da capacidade total da bacia. No intervalo de 535 a 635 m de altitude, há 3 reservatórios ou 1,2\% do total, e um volume máximo acumulável de $0,5 \mathrm{hm}^{3}$. Por último, a faixa de cota que varia de 635 a $735 \mathrm{~m}$, tem um total de 15 reservatórios, o equivalente a $6 \%$ do total de açudes na região, e um volume de $13,62 \mathrm{hm}^{3}$, ou aproximadamente $4 \%$ do volume armazenável de água.

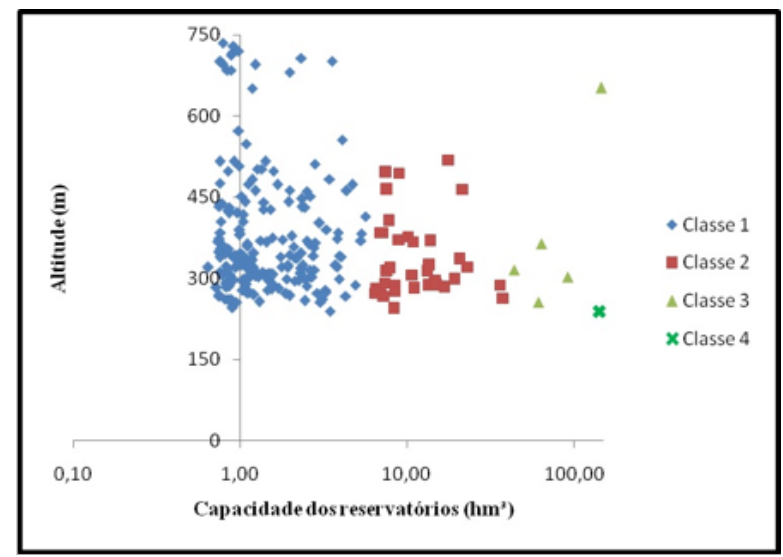

Figura 2: Relação entre a altitude e o porte dos reservatórios nas diferentes classes de capacidade de acumulação

No cenário em que existe apenas o reservatório estratégico Fogareiro, o qual se encontra no ponto de menor altitude da bacia, a disponibilidade hídrica é de 141 $\mathrm{hm}^{3}$ /ano com baixa oferta hídrica quando comparado, por exemplo, com o cenário real, em que os açudes estão distribuídos em diferentes cotas, e ofertam no total $227 \mathrm{hm}^{3} /$ ano. Esses, em sua maioria, são açudes de pequeno porte e estão classificados como classe 1. Vale ressaltar que o acúmulo de água em diversos reservatórios à montante do açude de maior porte contribui significativamente com o armazenamento de água na bacia. Tal fato pode ser observado a partir do cenário real, onde a disponibilidade hídrica em condições normais de acumulação de água no sistema é $60 \%$ maior que no cenário somente com o açude Fogareiro.

Outro fator importante é que a construção dos açudes em diferentes altitudes na bacia melhora a distribuição da água para as comunidades rurais mais remotas. Essa situação é observada na bacia hidrográfica do Fogareiro, na qual a elevada concentração de açudes em maiores altitudes (entre 300 e $350 \mathrm{~m}$ ) disponibiliza até pouco mais de $100 \mathrm{hm}^{3} / \mathrm{ano}$, em detrimento da água disponibilizada pelo açude Fogareiro na altitude 239 (Figura 3). Nos demais cenários avaliados, em que se avaliou um aumento da quantidade de açudes variando de $50 \%$ a $300 \%$, há uma tendência de aumento da disponibilidade hídrica nas cotas mais elevadas, apesar de a disponibilidade hídrica total do sistema não sofrer alteração significativa.

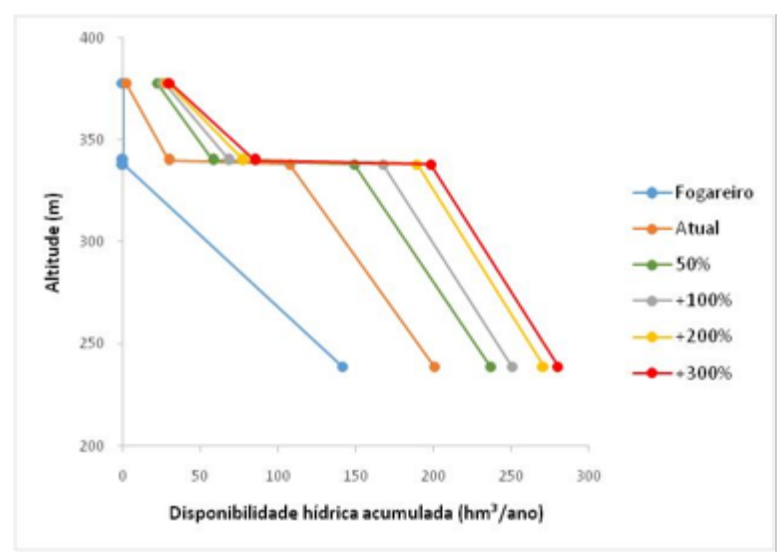

Figura 3: Relação entre a altitude e disponibilidade hídrica acumulada em diversos cenários de reservação na bacia hidrográfica do açude Fogareiro

A disponibilidade hídrica nos diferentes cenários mostra que a classe 1 (pontos em cotas mais elevadas na Figura 1, onde encontram-se predominantemente reservatórios de pequeno porte, possui maior quantidade de açudes. Entretanto, essa classe disponibiliza pouca água quando comparada, por exemplo, com as classes 2 e 3 em que a capacidade dos reservatórios é maior. Outro aspecto relevante é que essas classes são capazes de operar em um cenário de $+300 \%$ com melhor eficiência que a classe 1 , enquanto essa última sofre um decrescimento no rendimento a partir do cenário de $+100 \%$. Ao ponto que os reservatórios de médio porte melhoram a eficiência hidrológica, o reservatório Fogareiro permanece com vazão praticamente constante, o que significa dizer que o acréscimo de açudes na bacia provoca um impacto relativamente pequeno no reservatório estratégico.

Além de promover a disponibilidade hídrica de 
EFEITO DA REDE DE RESERVATÓRIOS SOBRE A ENERGIA HIDRÁULICA ACUMULADA EM BACIA HIDROGRÁFICA DE MESO-ESCALA NO SEMIÁRIDO BRASILEIRO

Tabela 1: Disponibilidade hídrica da bacia hidrográfica do açude Fogareiro nos diversos cenários. N: número de reservatórios; Barras cinza: disponibilidade hídrica

\begin{tabular}{|c|c|c|c|c|c|c|c|c|}
\hline \multirow[b]{2}{*}{ Cenário } & \multicolumn{2}{|c|}{ Classe 1} & \multicolumn{2}{|c|}{ Classe 2} & \multicolumn{2}{|c|}{ Classe 3} & \multicolumn{2}{|c|}{ Classe 4} \\
\hline & $\mathbf{N}$ & $\begin{array}{c}\text { Vazão } \\
\left(\mathbf{h m}^{3} / \mathbf{a n o}\right)\end{array}$ & $\mathbf{N}$ & $\begin{array}{c}\text { Vazão } \\
\left(\mathrm{hm}^{3} / \mathbf{a n o}\right)\end{array}$ & $\mathbf{N}$ & $\begin{array}{c}\text { Vazão } \\
\text { (hm'/ano) }\end{array}$ & $\mathbf{N}$ & $\begin{array}{c}\text { Vazão } \\
\left(\mathrm{hm}^{3} / \mathbf{a n o}\right)\end{array}$ \\
\hline Fogareiro & 0 & 0,00 & 0 & 0,00 & $\overline{0}$ & 0,00 & 1 & 141,28 \\
\hline Atual & 195 & 16,68 & 35 & 29,82 & 10 & 71,06 & 1 & 92,44 \\
\hline$+50 \%$ & 293 & 21,69 & 53 & 39,33 & 15 & 83,52 & 2 & 87,97 \\
\hline$+100 \%$ & 390 & 25,99 & 70 & 45,58 & 20 & 91,41 & 1 & 83,32 \\
\hline$+300 \%$ & 780 & 29,91 & 140 & 58,28 & 40 & 106,97 & 1 & 81,74 \\
\hline
\end{tabular}

forma mais uniformemente distribuída na bacia, a presença de pequenos reservatórios em cotas mais altas também minimiza os gastos com energia para bombeamento da água dos reservatórios de jusante para população residente a montante (DE ARAÚJO; MEDEIROS 2013). Essa eficiência hidráulica é possível devido à redução da altura geométrica $(\mathrm{Hg})$, ou seja, aquela que precisa ser vencida pelo sistema de bombeamento (Figura (4). Nesse sentido, a partir da aproximação dos reservatórios aos centros de demanda hídrica, o sistema tende a apresentar maior eficiência hidrológica e energética, visto que, caso não houvesse os pequenos e médios açudes, a água armazenada apresentaria cotas mais baixas nos reservatórios estratégicos de jusante, demandando mais energia para disponibilizá-la para as comunidades a montante.

\section{CONCLUSÕES}

As simulações de disponibilidade hídrica na bacia do açude Fogareiro em diferentes cenários de armazenamento de água permitem concluir que a grande quantidade de pequenos açudes resulta em uma oferta hídrica relativamente pequena se comparada com aquela promovida por açudes de maior porte. Entretanto, tem-se um ganho relacionado à distribuição espacial da água na bacia. Além disso, a introdução de reservatórios de pequeno porte próximos das cabeceiras da bacia, e, portanto em cotas mais elevadas, promove um aumento de altitude média no que concerne à disponibilidade hídrica do sistema, resultando em um ganho de energia hidráulica potencial. Assim, a distribuição de água para comunidades remotas a partir de reservatórios próximos e em cotas mais elevadas tende a reduzir os gastos energéticos se comparados a sistemas com poucos reservatórios de pequeno porte e concentração da disponibilidade hídrica em reservatórios estratégicos, os quais geralmente se encontram mais a jusante e em altitudes mais baixas.

\section{AGRADECIMENTOS}

Os autores agradecem à FUNCAP e ao CNPq pela concessão de bolsas de mestrado e iniciação científica ao primeiro e segundo autores, respectivamente, e ao $\mathrm{CNPq}$ pelo apoio financeiro no âmbito do projeto "Gestão de pequenos açudes no semiárido - GEPASA" (455883/2014-9).

\section{REFERÊNCIAS}

ADHAM, A.; RIKSEN, M.; OUESSAR, M.; RITSEMA, C. Identification of suitable sites for rainwater harvesting structures in arid and semi-arid regions: A review. International Soil and Water Conservation Research, v. 4, n. 2, p. 108 - 120, 2016.

ALBUQUERQUE, A. A.; FIRMINO, M. B. M.; CURI, W. F. Uso racional de energia no bombeamento de água em sistema de abastecimento via programação não-linear. Seminário Hispano-Brasileiro sobre Sistemas de Abastecimento Urbano de Água, João Pessoa, 2004.

ANDRADE, E.; PEREIRA, O.; DANTAS, F. Semiárido e o manejo adequado do capital natural. Il. Color., p. 408, 2010.

BRASIL. Plano Nacional de Eficiência Energética: premissas e diretrizes básicas. Brasília: Ministério de Minas e Energia, Secretaria de Planejamento e Desenvolvimento Energético, 2011.

CALLOW, J.; SMETTEM, K. The effect of farm dams and constructed banks on hydrologic connectivity and runoff estimation in agricultural landscapes. Environmental Modelling \& Software, v. 24, n. 8, p. 959-968, 2009.

DE ARAÚJO, J. C.; GÜNTNER, A.; BRONSTERT, A. Loss of reservoir volume by sediment deposition 


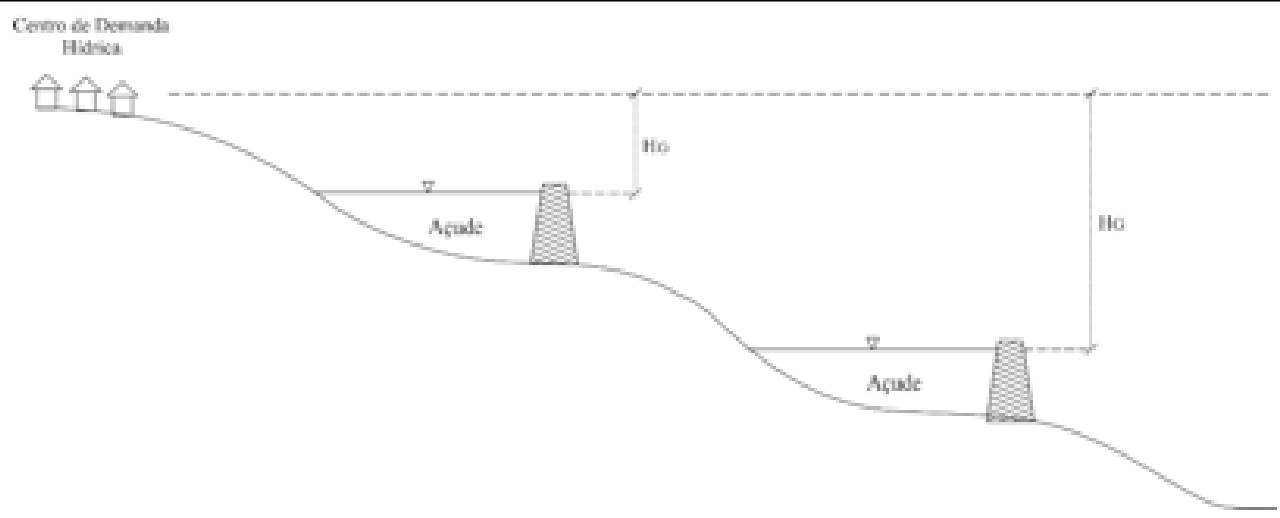

Figura 4: Representação da redução da altura geométrica e da eficiência energética no sistema

and its impact on water availability in semiarid brazil. Hydrological Sciences Journal, v. 51, n. 1, p. 157-170, 2006.

DE ARAÚJO, J. C.; MEDEIROS, P. H. A. Impact of dense reservoir networks on water resources in semiarid environments. Australasian Journal of Water Resources, v. 17, n. 1, p. 87-100, 2013.

DE ARAÚJO, J. C.; PIEDRA, J. I. G. Comparative hydrology: analysis of a semiarid and a humid tropical watershed. v. 23, n. 8, p. 1169-1178, 2009.

DOS SANTOS, M. J.; ARAÚJO, L. E.; OLIVEIRA, E. M.; SILVA, B. B. Seca, precipitação e captação de água de chuva no semiárido de sergipe. Engenharia Ambiental, Espírito Santo do Pinhal, v. 6, p. 055 - 073, 2009.

FERREIRA, J. H. I.; CAMACHO, J. R.; MALAGOLI, J. A.; GUIMARÃES JÚNIOR, S. C. Assessment of the potential of small hydropower development in brazil. Renewable and Sustainable Energy Reviews, v. 56, p. 380 - 387, 2016.

FOWE, T.; KARAMBIRI, H.; PATUREL, J.; POUSSIN, J.; CECCHI, P. Water balance of small reservoirs in the volta basin: A case study of boura reservoir in burkina faso. Agricultural Water Management, v. 152, p. 99 - 109, 2015.

\section{FUNDAÇÃO CEARENSE DE METEOROLOGIA E RECURSOS HÍDRICOS. Mapeamento dos} espelhos d'água do Brasil. Ceará: FUNCEME, 2008. Disponível em: <http://www.funceme.br/documents/ Projetos/espelhos_dagua.pdf $>$. Acesso em: 22 set. 2016.
GAISER, T.; KROL, M. S.; FRISCHKORN, H.; DE ARAÚJO, J. C. Global change and regional impacts: Water availability and vulnerability of ecosystems and society in the semi-arid northeast of brazil. Berlin, 2003.

GÜNTNER, A.; S., K. M.; ARAÚJO, J. C. D.; BRONSTERT, A. Simple water balance modelling of surface reservoir systems in a large data-scarce semiarid region / modélisation simple du bilan hydrologique de systèmes de réservoirs de surface dans une grande région semi-aride pauvre en données. Hydrological Sciences Journal, v. 49, n. 5, p. 901-918, 2004.

KROL, M. S.; DE VRIES, M. J.; VAN OEL, P. R.; DE ARAÚJO, J. C. Sustainability of small reservoirs and large scale water availability under current conditions and climate change. Water Resources Management, v. 25 , n. 12, p. 3017 - 3026, 2011.

LIEBE, J. R.; VAN DE GIESEN, N.; ANDREINI, M.; WALTER, M. T.; STEENHUIS, T. S. Determining watershed response in data poor environments with remotely sensed small reservoirs as runoff gauges. Water Resources Research, v. 45, 2009.

LIMA NETO, I. E.; WIEGAND, M. C.; ARAÚJO, J. C. D. Sediment redistribution due to a dense reservoir network in a large semi-arid brazilian basin. Hydrological Sciences Journal, v. 56, n. 2, p. 319-333, 2011.

MALVEIRA, V. T. C.; ARAÚJO, J. C.; GÜNTNER, A. Hydrological impact of a high- density reservoir network in the semiarid north-eastern brazil. Journal of Hydrologic Engineering, v. 17, n. 1, p. 109-117, 2012. 
PAIVA, M. P. Trabalhos esparsos, agora reunidos. Edições Livro Técnico, Fortaleza, p. 15-33, 2008.

PEREIRA, B. S. Caracterização geométrica de pequenos açudes no semiárido cearense. Monografia (Graduação em Engenharia Ambiental e Sanitária), Departamento de Meio Ambiente, IFCE-Maracanaú, p. 51, 2014.

PETER, S. J.; ARAÚJO, J. C. D.; ARAÚJO, N. A. M.; HERRMANN, H. J. Flood avalanches in a semiarid basin with a dense reservoir network. Journal of Hydrology, v. 512, p. 408-420, 2014.

PROSAB. Conservação de água e energia em sistemas prediais e públicos de abastecimento de água. Ricardo Francisco Gonçalves (coord.). Rio de Janeiro: ABES, 2009.

ROCHA, R.; SOARES, R. Water scarcity and birth outcomes in the brazilian semiarid. Journal of Development Economics, v. 112, p. 72 - 91, 2015.

SILANS, A. M. B. P. Redução da evaporação de açudes - o estado da arte. Revista Brasileira de Recursos Hídricos, v. 8, n. 2, p. 101-109, 2003. 